\title{
Flora fúngica no ambiente da Unidade de Terapia Intensiva Pediátrica e Neonatal em hospital terciário
}

\author{
Environmental fungal flora in Pediatric and Neonatal Intensive Care Units at a tertiary hospital
}

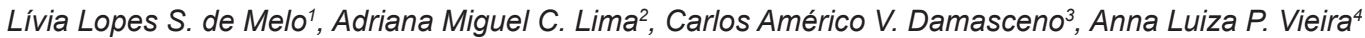

\section{RESUMO}

Objetivo: As infecções nosocomiais são responsáveis por morbidade e mortalidade significativas no período neonatal. Considerando-se a preocupação com a qualidade do ar de áreas críticas como Unidades de Terapia Intensiva (UTI), foi realizado um levantamento da flora fúngica das UTI Pediátrica e Neonatal do Hospital das Clínicas Samuel Libânio, Pouso Alegre (MG), com a finalidade de identificar a presença de fungos potencialmente patogênicos e oportunistas.

Métodos: Foram realizadas 30 coletas, que incluíram leitos, incubadoras, janelas, aparelhos de ar condicionado, telefone, estetoscópios, portas e maçanetas. Placas de Agar Sabouraud Dextrose com o material das coletas foram incubadas em temperatura ambiente por 15 dias. A identificação foi baseada nas características macroscópicas no exame direto e em microcultivos.

Resultados: Fungos potencialmente patogênicos e toxigênicos foram isolados. A análise quantitativa das colônias revelou a presença de 11 gêneros. Verificou-se que mais de $40 \%$ das colônias correspondem ao gênero Penicillium spp, seguido por Cladosporium spp e Chrysosporium spp.

Conclusões: Os fungos encontrados podem apresentar grande potencial de patogenicidade, principalmente em imunodeprimidos. É importante adotar medidas de controle ambiental, como assepsia dos equipamentos, controle da presença de visitantes, lavagem das mãos pelos funcionários e troca de filtros de ar condicionado.

Palavras-chave: fungos; infecção hospitalar; infecções fúngicas; unidades de terapia intensiva pediátrica; unidades de terapia intensiva neonatal.

\section{ABSTRACT}

Objective: Nosocomial infections lead to significant morbidity and mortality in the neonatal period. Considering the concern regarding air quality in critical hospital areas, such as Intensive Care Units (ICU), this study aims to identify the presence of potentially pathological fungi in the Pediatric and Neonatal Intensive Care Unit of the Samuel Libânio Hospital in Pouso Alegre, Minas Gerais, Brazil.

Methods: Thirty samples were collected in the following areas: surface of beds, incubators, windows, air conditioning, telephones, stethoscopes, doors and door knobs. Petri dishes of Sabouraud Dextrose Agar with the collected samples were incubated at air temperature for 15 days. The identification was based on macroscopic and microscopic characteristics and subcultures.

Results: Potentially pathological and toxigenic fungi were isolated in the studied areas. The quantitative analysis revealed the presence of 11 genera: $40 \%$ of the colonies were Penicillium spp., followed by Cladosporium spp. and Chrysosporium spp.

Conclusions: The fungi found may present a great pathogenic potential, mainly in immunocompromised patients. It is important to adopt some measures in order to reduce nosocomial infection in ICU, such as the strict control regarding equipment cleansing, control of visitors, hand washing and periodic cleansing and renewal of air conditioning filters.

Key-words: fungi; cross infection; mycoses; intensive care units, pediatric; intensive care units, neonatal.
Instituição: Universidade do Vale do Sapucaí (Univas), Pouso Alegre, MG, Brasil

${ }^{1}$ Aluna do curso de Medicina da Univas; Bolsista da Fundação de Amparo à Pesquisa do Estado de Minas Gerais (Fapemig), Pouso Alegre, MG, Brasil ${ }^{2}$ Aluna do curso de Medicina da Univas, Pouso Alegre, MG, Brasil 32Doutor em Ciências Biológicas pela Universidade Federal de Minas Gerais; Professor adjunto da UFMG; Professor titular de Microbiologia e Imunologia da Univas, Pouso Alegre, MG, Brasil

${ }^{4}$ Mestre em Saúde pela Universidade Federal de São Paulo; Médica assistente da Unifesp; Professora adjunta da Disciplina de Pediatria da Univas, Pouso Alegre, MG, Brasil
Endereço para correspondência:

Lívia Lopes S. de Melo

Avenida Nove de Julho, 3.239/101 - Jardim Paulista CEP 01407-000 - São Paulo/SP

E-mail: livialmelo@hotmail.com

Fonte financiadora: Fundação de Amparo à Pesquisa do Estado de Minas Gerais (Fapemig), processo n50034/07.

Recebido em: 10/8/08

Aprovado em: 15/2/09 


\section{Introdução}

As infecções nosocomiais são responsáveis por morbidade e mortalidade significativas no período neonatal. As unidades de terapia intensiva pediátrica e neonatal são consideradas áreas críticas, tento em vista a realização de grande quantidade de procedimentos invasivos e a gravidade das crianças internadas. Dentre as infecções frequentes em crianças internadas em Unidades de Terapia Intensiva (UTI), há preocupação especial com as fúngicas. Devido à imaturidade do sistema imune do recém-nascido e ao uso de antibioticoterapia de largo espectro, fungos comumente encontrados no ar podem se tornar patógenos ${ }^{(1-3)}$. Nas imunodeficiências, em crianças mais velhas, também se criam condições para numerosas infecções por fungos ${ }^{(4-6)}$. Desta forma, assume grande importância o estudo dos fungos oportunistas, especialmente em ambiente hospitalar.

Em um dos poucos estudos sobre a flora fúngica em UTI Pediátrica e Neonatal, Martins-Diniz et a $l^{(7)}$ descreveram o gênero Cladosporium spp como o principal isolado, seguido por Fusarium spp, Penicillium spp, Chrysosporium spp e Aspergillus spp. Em amostras colhidas do mobiliário, a Candida albicans mostrou-se frequente. Em estudo anterior, Silva et $a l^{(8)}$ haviam destacado a elevada frequência de Cladosporium spp, seguidos por Aspergillus spp, Fusarium spp, Penicillium spp e Aureobasidium spp.

Mobin et $a l^{(9)}$, em uma pesquisa da microbiota fúngica em condicionadores de ar, descreveram o gênero Aspergillus spp como predominante. Sistemas de ar condicionado contaminados já foram implicados em infecções por fungos como Aspergillus spp e Rhizopus spp ${ }^{(4)}$.

Considerando a preocupação com a qualidade do ar interno de ambientes hospitalares como as Unidades de Terapia Intensiva, realizou-se um levantamento da flora fúngica em UTI Pediátrica e Neonatal, com o objetivo de identificar a presença de fungos potencialmente patogênicos e oportunistas.

\section{Métodos}

Foi realizado um estudo transversal na UTI Pediátrica e Neonatal do Hospital das Clinicas Samuel Libânio, na cidade de Pouso Alegre (MG). Na entrada da área, há duas portas que separam área externa, recepção e UTI. Esta contém nove leitos, três pediátricos e seis neonatais, sendo um de isolamento. A divisão dos leitos no espaço físico está de acordo com as normas exigidas e a unidade é aprovada pela Vigilância Sanitária. Em duas paredes estão distribuídas seis janelas, que permanecem fechadas enquanto os dois aparelhos de ar condicionado estão ligados, o que eventualmente não ocorre nos períodos de inverno. Todos os leitos são equipados com monitores, oxímetros e respiradores. Ainda fazem parte do local: quarto e banheiro dos funcionários, sala de expurgo, depósito de material de limpeza, banheiro para os pacientes, posto de enfermagem, pia para lavagem das mãos e uma mesa onde ficam os prontuários e um aparelho telefônico. A limpeza de todo o local ocorre no período das 7 às 17 horas, todos os dias da semana. A equipe de funcionários conta com quatro técnicos de enfermagem, um enfermeiro, um médico de plantão 24 horas e outro no período da manhã. Cada leito possui estetoscópio próprio.

Durante o ano de 2007, houve um total de 222 internações. Destas, $159(71 \%)$ foram neonatais e $63(28,5 \%)$ pediátricas. As internações neonatais foram decorrentes de desconforto respiratório precoce (47\%), anomalias congênitas (15\%), muito baixo peso (21\%), baixo peso extremo (9\%), sepse neonatal $(5 \%)$ e asfixia (3\%). As internações pediátricas foram decorrentes de patologias neurológicas (30\%), cardiopatias congênitas (20\%), sepse (17\%), síndrome da angústia respiratória aguda (17\%), politrauma (10\%) e outras (6\%). Entre o primeiro e o segundo dia de vida, em geral, é colocado um cateter central de inserção periférica nos pacientes neonatais para a nutrição parenteral. O esquema de antibiótico utilizado para o tratamento de sepse neonatal precoce é ampicilina e gentamicina e, para sepse tardia, oxacilina e aminoglicosídeos.

A incidência de sepse no setor é de aproximadamente sete casos por mil pacientes/dia, sendo que $20 \%$ desses casos se associam à infecção fúngica. Semanalmente, é realizada a pesquisa de fungos na urina de pacientes crônicos em tratamento com nutrição parenteral e antibióticos, após duas semanas de internação.

A coleta do material dos leitos, incubadoras, janelas, aparelhos de ar condicionado, telefones, estetoscópios, portas e maçanetas foi realizada no período de abril a dezembro de 2007, com um total de 30 coletas distribuídas em três ou quatro por mês. O período do dia escolhido foi o final da tarde, por ser após o período de visitas e devido ao maior potencial de contaminação do local.

Para a coleta do material do ar, foram utilizadas 17 placas de Petri com Agar Sabouraud Dextrose (Himedia ${ }^{\circledR}$ ), abertas durante 30 minutos, em lugares próximos aos locais estudados. Foram distribuídas seis placas nas janelas, nove nos leitos e incubadoras e duas nas saídas do ar condicionado. Outras 
13 amostras foram recolhidas com swab estéril e semeadas no Agar Sabouraud. Destas, duas foram colhidas dos aparelhos de ar condicionado, uma da superfície do telefone, quatro dos estetoscópios e as outras cinco das portas e maçanetas. As 30 placas foram incubadas em temperatura ambiente por aproximadamente $15 \operatorname{dias}^{(10)}$ e, a partir de então, iniciou-se a identificação dos fungos.

Após o crescimento da cultura primária, em um primeiro momento foi feita a análise macroscópica, iniciando a identificação do gênero baseada nas características morfológicas de cada colônia, como forma e cor. Associaram-se, então, as características microscópicas do exame direto, processo primariamente apoiado nas diferenças morfológicas das estruturas reprodutivas e no modo como os esporos são produzidos ${ }^{(11)}$. Posteriormente, para o melhor desenvolvimento dessas estruturas reprodutivas, foram montadas microculturas para confirmar a identificação. Após o crescimento, os microcultivos foram montados em lâminas, corados e submetidos à inspeção microscópica, a qual permitiu uma visualização melhor dos elementos pela manutenção da integridade das estruturas microscópicas ${ }^{(12)}$.

\section{Resultados}

Fungos potencialmente patogênicos e toxigênicos foram isolados nos locais estudados na UTI Pediátrica e Neonatal do Hospital das Clinicas Samuel Libânio no período de abril a dezembro de 2007. A análise quantitativa das colônias, considerando-se todos os ambientes estudados, revelou a presença de
11 gêneros, com frequência variável de Penicillium spp, Cladosporium spp, Chrysosporium spp, Aspergillus spp, Exserobilum spp, Aureobasidium spp, Curvularia spp, Alternaria spp, Scopulariopsis spp, Rhizopus spp e Bipolaris spp (Tabela 1, Figura 1).

Nas 30 placas expostas, houve crescimento de 160 colônias, contadas em Unidades Formadoras de Colônia (UFC). Verificou-se que mais de $40 \%$ das colônias correspondiam ao gênero Penicillium spp, seguido por Cladosporium spp e Chrysosporium spp (Gráfico 1). Entre os gêneros mais freqüentes, Cladosporium spp foi isolado em todos os lugares estudados, enquanto Penicillium spp e Chrysosporium spp só não estiveram presentes no aparelho de telefone.

Das colônias isoladas com menos frequência, destaca-se a presença de Aspergillus spp e Curvularia spp nos aparelhos de ar condicionado, de Aureobasidium spp, Exserobilium spp, Alternaria spp e Curvularia spp nas janelas, de Bipolaris spp nas incubadoras, Scopulariopsis spp e Rhizopus spp nas portas e maçanetas.

Das 30 placas, vinte e sete se mostraram positivas para a presença de leveduras. Das outras três placas nas quais não houve crescimento de colônias, duas haviam sido expostas ao ar de duas incubadoras e uma fez parte da coleta por swab da parte interna da porta de entrada.

\section{Discussão}

O número de estudos que abordam a contaminação fúngica em UTI Pediátrica e Neonatal é bastante reduzido ${ }^{(2,3)}$, mas apresenta grande importância devido ao aparecimento de agentes fúngicos em infecções nosocomiais.

Tabela 1 - Unidades formadoras de colônias de fungos isolados na UTI Pediátrica e Neonatal do Hospital das Clínicas Samuel Libânio

\begin{tabular}{|c|c|c|c|c|c|c|c|}
\hline Gênero & Janelas & $\begin{array}{c}\mathrm{Ar} \\
\text { condicionado }\end{array}$ & $\begin{array}{l}\text { Incubadoral } \\
\text { leitos }\end{array}$ & $\begin{array}{c}\text { Portas/ } \\
\text { maçanetas }\end{array}$ & Telefone & Estetoscópio & Total \\
\hline Penicillium spp. & 49 & 1 & 13 & 3 & - & 1 & 67 \\
\hline Cladosporium spp. & 18 & 4 & 21 & 5 & 1 & 1 & 50 \\
\hline Chrysosporium spp. & 14 & 4 & 2 & 2 & - & 1 & 23 \\
\hline Aspergillus spp. & 1 & 2 & - & 1 & - & - & 4 \\
\hline Exserohilum spp. & 3 & - & 1 & - & - & - & 4 \\
\hline Aureobasidium spp. & 3 & - & - & - & - & - & 3 \\
\hline Curvularia spp. & 1 & 1 & - & - & - & - & 2 \\
\hline Alternaria spp. & 2 & - & - & - & - & - & 2 \\
\hline Scopulariopsis spp. & - & - & - & 2 & - & - & 2 \\
\hline Rhizopus spp. & - & - & - & 2 & - & - & 2 \\
\hline Bipolaris spp. & - & - & 1 & - & - & - & 1 \\
\hline Total & & & & & & & 160 \\
\hline
\end{tabular}



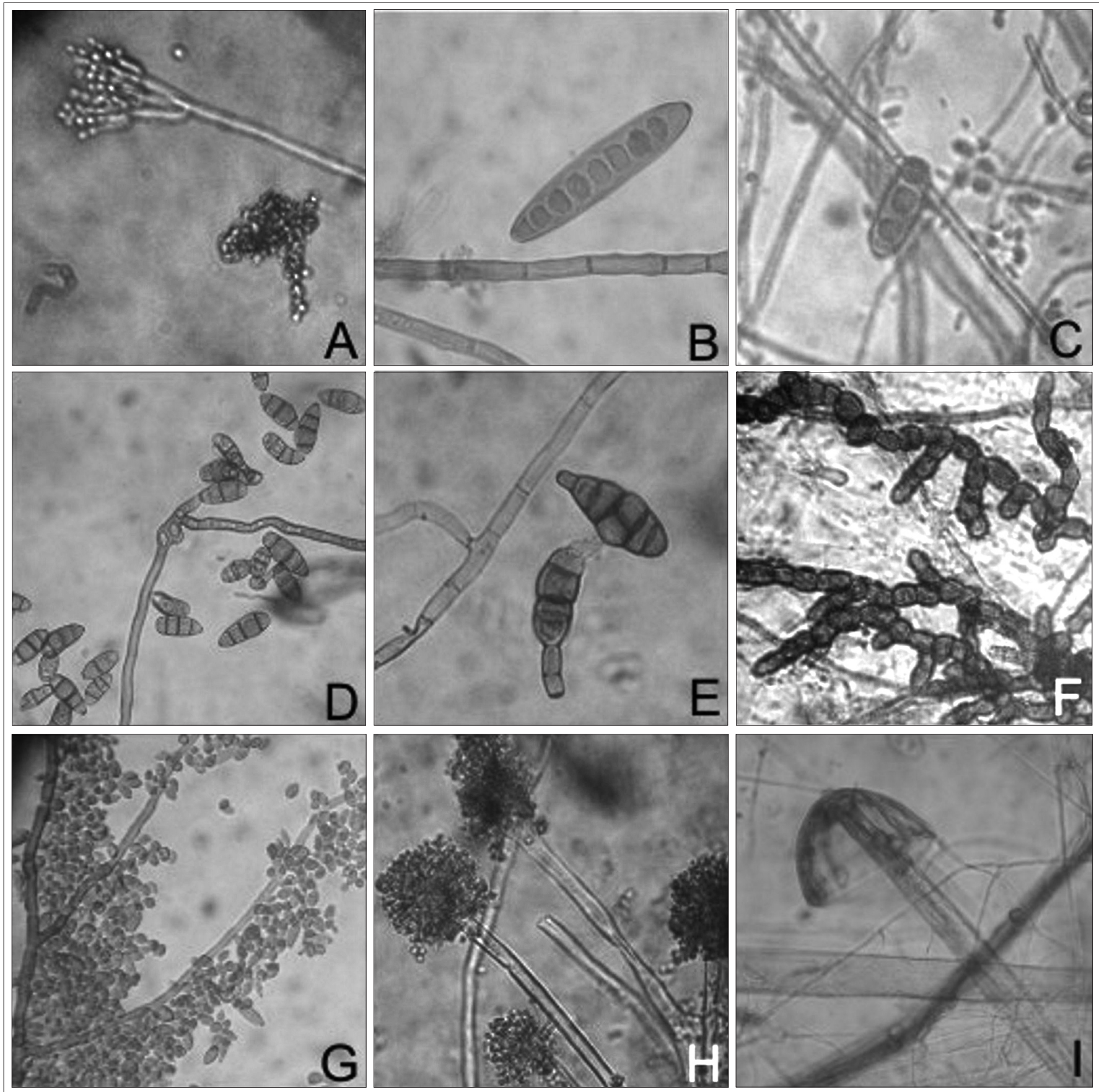

Figura 1 - Exame direto de alguns dos fungos isolados e corados com Lactofenol: (A) Penicillium spp. (B) Exserohilum spp. (C) Bipolaris spp. (D) Curvularia spp. (E) Alternaria spp. (F) Aureobasidium spp. (G) Cladosporium spp. (H) Aspergillus spp. (I) Rhizopus spp.

Os resultados encontrados nesta pesquisa, em geral, são concordantes com os de outros estudos. A prevalência dos gêneros Penicillium spp, Cladosporium spp e Chrysosporium spp em UTI Pediátrica e Neonatal também foi descrita por Martins-Diniz et al ${ }^{(7)} \mathrm{em}$ um estudo de monitoramento de fungos em unidade hospitalar. Silva et al ${ }^{(8)}$ também observa- ram frequência elevada de Cladosporium spp na investigação de todo o ambiente hospitalar.

Os gêneros isolados em menor frequência mostraram discordâncias em diferentes estudos. Martins-Diniz et al $l^{(7)}$ e Silva et $a^{(8)}$ descreveram Aspergillus spp e Fusarium spp entre os gêneros mais frequentes. No presente estudo, o 
Aspergillus spp foi isolado com baixa frequência e o Fusarium spp não foi identificado em nenhuma cultura. A variação na frequência desses fungos pode ser explicada pelos diferentes locais de exposição, técnicas de coleta, temperatura, umidade do ar, estações do ano e atividade humana ${ }^{(13-18)}$. De qualquer maneira, os fungos encontrados podem apresentar grande potencial de patogenicidade, principalmente em pacientes imunodeprimidos.

Penicillium spp, o gênero mais frequente, isolado em todos os locais estudados, com exceção do aparelho de telefone, geralmente não é patogênico para o homem ${ }^{(14)}$. Algumas espécies, entretanto, já foram descritas como causadoras de reações alérgicas e infecções sistêmicas em imunodeprimidos ${ }^{(19)}$. Cladosporium spp, isolado em todos os locais, é agente de lesões cerebrais e cutâneas, abscessos cerebrais acompanhados ou não de meningite e cromomicose ${ }^{(14)}$. Chrysosporium spp, só ausente no aparelho de telefone, causa lesões ungueais ${ }^{(14)}$.

Aspergillus spp, encontrado nos aparelhos de ar condicionado, é um agente oportunista por excelência. Várias espécies podem provocar "colonização" em cavidades pré-existentes (aspergiloma intracavitário), infecções propriamente ditas, processos alérgicos e intoxicações (micotoxicoses). As formas clínicas mais comumente observadas são a pulmonar, a cutânea (cistos) e a orbitária ${ }^{(20)}$. São relatados como agentes etiológicos de osteomielite fúngica vertebral em pacientes submetidos a procedimentos invasivos ${ }^{(21)}$ e também como importante agentes de otomicoses $^{(22)}$. A aspergilose invasiva teve seu aumento assinalado nos últimos anos, principalmente em pacientes imunocomprometidos ${ }^{(23-27)}$.

Exserobilum spp, encontrado nas janelas e incubadoras, leva à feo-hifomicose, com lesões cutâneas e osteomusculares, sinusite e infecções disseminadas ${ }^{(14)}$. Já Aureobasidium spp, encontrado nas janelas, é um fungo oportunista de infecções generalizadas ou sistêmicas, abscessos em diversas vísceras ${ }^{(28)}$ e quadros de fungemia ${ }^{(29)}$. Curvularia spp, isolado nas janelas e aparelhos de ar condicionado, é agente de ceratite, infecções disseminadas, sinusite invasiva, lesões cutâneas e osteolíticas ${ }^{(14)}$. Há relato de um caso de peritonite $^{(30)}$.

Alternaria spp, presente nas janelas, é oportunista por excelência e tem sido isolado em lesões cutâneas, osteolíticas e onicomicoses em pacientes imunodeprimidos ${ }^{(14)}$. Scopulariopsis spp, isolado de portas e maçanetas, representa um dos agentes mais frequentes de onicomicoses e também de pneumonia e lesões cutâneas ${ }^{(14)}$. Finalmente, Rhizopus spp,

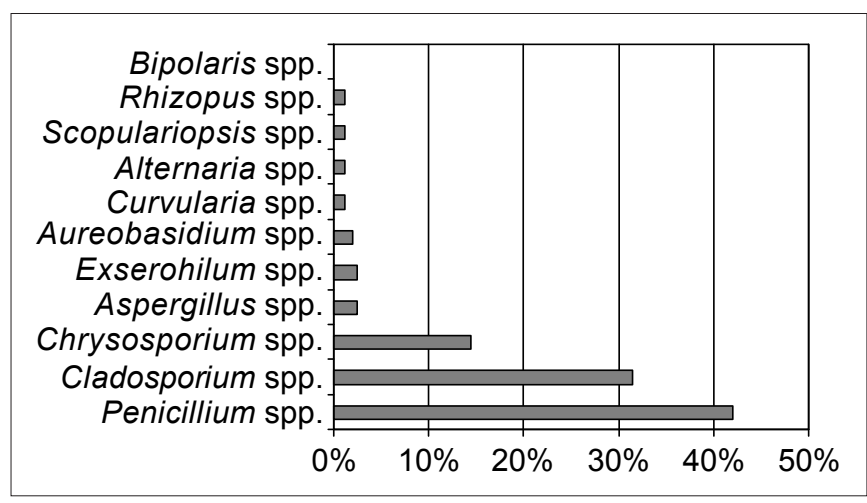

Gráfico 1 - Gêneros dos fungos encontrados na UTI Pediátrica e Neonatal do Hospital das Clínicas Samuel Libânio.

também isolado em portas e maçanetas, é o agente encontrado com maior frequência em mucormicoses ${ }^{(29)}$ e o Bipolaris spp, isolado em uma das incubadoras, leva a quadros diversos de meningoencefalite, sinusite, lesões ósseas, cutâneas e broncopulmonares ${ }^{(14)}$.

Não houve, durante o período de estudo, associação entre a clínica dos pacientes da UTI Pediátrica e Neonatal com as possíveis doenças causadas pelos fungos descritos. Porém, ocorreram quatro episódios de onicomicoses em funcionários do setor.

O monitoramento da microbiota fúngica dos aparelhos de ar condicionado foi um dos pontos de maior relevância do presente estudo. Os resultados encontrados, como o isolamento do gênero Aspergillus spp, são preocupantes, mesmo em baixa incidência, pois esse é o gênero que apresenta a maior patogenicidade dentre os isolados.

É importante que, a partir desses resultados, se façam orientações de medidas preventivas. Os profissionais que trabalham em Unidades de Terapia Intensiva devem ser sensibilizados para a existência de infecções fúngicas e determinar a limpeza adequada dos condicionadores de $\mathrm{ar}^{(9)}$. Na unidade avaliada, essas orientações foram feitas e se estabeleceu uma nova rotina de limpeza dos aparelhos.

A presença de outros fungos patogênicos em todos os locais estudados reforça a importância de medidas de controle de infecção hospitalar em UTI, como cuidados referentes à técnica de assepsia dos equipamentos, controle da presença de visitantes e lavagem das mãos pelos funcionários.

Dessa forma, a caracterização dos fungos de áreas críticas de hospitais como a UTI Pediátrica e Neonatal é uma importante ação e, se realizada de acordo com as orientações de medidas para o controle dos potenciais patógenos encontrados nos locais monitorizados, pode contribuir para diminuir a morbidade e a mortalidade decorrentes de infecções nosocomiais. 


\section{Referências bibliográficas}

1. Behrman RE, Kliegman RM, Jenson HB. Nelson: tratado de pediatria. $17^{\mathrm{a}}$ ed. Rio de Janeiro: Elsevier; 2005.

2. David CM. Infecção em UTI. Medicina, Ribeirão Preto 1998;31:337-48.

3. Horn WA, Larson EL, McGinley KJ, Leyden JJ. Microbial flora on the hands of health care personnel: differences in composition and antibacterial resistance. Infect Control Hosp Epidemiol 1988;9:189-93.

4. Morais MB, Campos SO, Silvestrini WS. Guia de pediatria. Barueri: Manole; 2005.

5. Lacaz CS, Porto E, Martins JE, Heins-Vaccari EM, Melo NT. Tratado de micologia médica Lacaz. 9a ed. São Paulo: Sarvier; 2002.

6. Mousa HA, Al-Bader SM, Hassan DA. Correlation between fungi isolated from burn wounds and burn care units. Burns 1999;25:145-7.

7. Martins-Diniz JN, Silva RA, Miranda ET, Mendes-Giannini MJ. Monitoring of airborne fungus and yeast species in a hospital unit. Rev Saude Publica 2005;39:398-405.

8. Silva MG, Moreira YK, Cisalpino EO. Flora fúngica do ar e do piso no Hospital das Clínicas da Universidade de Minas Gerais, Belo Horizonte, Brasil. Rev Microbiol 1983;14:215-22.

9. Mobin M, Salmito MA. Microbiota fúngica dos condicionadores de ar nas unidades de terapia intensiva de Teresina, PI. Rev Soc Bras Med Trop 2006;39:556-9.

10. Oliveira JC. Micologia médica. Rio de Janeiro: Ed Control Lab; 1999.

11. Zaitz C, Campbel I, Marques SA. Compêndio de micologia médica. Rio de Janeiro: Medsi; 1998.

12. Neufeld PM. Manual de micologia médica: técnicas básicas de diagnóstico. Rio de Janeiro: PNCQ; 1999.

13. Gambale W, Purchio A, Paula CR. Influência de fatores abióticos na dispersão aérea de fungos na cidade de São Paulo. Rev Microbiol 1983;14:204-14.

14. Lacaz CS, Porto E, Heins-Vaccari EM. Guia para identificação: fungos, actinomicetos e algas de interesse médico. São Paulo: Sarvier; 1998.

15. Nessa D, Lortholary J, Bouakline A, Bordes M, Chandenier J, Derouin F et al. Comparative performance of impactor air samplers for quantification of fungal contamination. J Hosp Infect 2001;47:149-55.

16. Overberger PA, Wadowsky RM, Schaper MM. Evaluation of airborne particulates and fungi during hospital renovation. Am Ind Hyg Assoc J 1995;56:706-12.

17. Távora LG, Gambale W, Heins-Vaccari EM, Arriagada GL, Lacaz CS, Santos
CR et al. Comparative performance of two air samplers for monitoring airborne fungal propagules. Braz J Med Biol Res 2003;36:613-6.

18. Mezzari A, Perin C, Santos Jr SA, Bernd LA, Gesu GD. Os fungos anemófilos e sensibilização em indivíduos atópicos em Porto Alegre, RS. Rev Assoc Med Bras 2003;49:270-3.

19. Noritomi DT, Bub GL, Beer I, da Silva AS, de Cleva R, Gama-Rodrigues JJ. Multiple brain abscesses due to Penicillium spp infection. Rev Inst Med Trop Sao Paulo 2005;47:167-70.

20. Lacaz CS, Porto E, Martins JE. Micologia médica: fungos, actinomicetos e algas de interesse médico. $8^{a}$ ed. São Paulo: Sarvier; 1991.

21. Figueiredo GC, Figueiredo EC, Tavares-Neto J. Clinical and therapeutic features of fungal vertebral osteomyelitis. A secondary data analysis. Rev Bras Reumatol 2007;47:34-41.

22. Zaror L, Fischman O, Suzuki FA, Felipe RG. Otomicose em São Paulo (Brasil). Rev Inst Med Trop S Paulo 1991;33:169-73.

23. Alberti C, Bouakline A, Ribaud P, Lacroix C, Rousselot P, Leblanc $T$ et al. Relationship between environmental fungal contamination and the incidence of invasive aspergillosis in haematogy patients. J Hosp Infect 2001;48:198-206.

24. Cooper EE, O'Reilly MA, Dharmage SC. Influence of building construction work on Aspergillus infection in a hospital setting. Infect Control and Hosp Epidemiol 2003;24:472-6.

25. Warris A, Verweij PE. Clinical implications of environmental sources for Aspergillus. Med Mycol 2005;43 (Suppl 1):S59-65.

26. Anaissie EJ, Stratton SL, Dignani MC, Lee CK, Summerbell RC, Rex JH et al. Pathogenic molds (including Aspergillus species) in hospital water distribution systems: a 3-year prospective study and clinical implications for patients with hematologic malignancies. Blood 2003;101:2542-6.

27. Sherertz RJ, Belani A, Kramer BS, Elfenbein GJ, Weiner RS, Sullivan ML et al. Impact of air filtration on nosocomial Aspergillus infections. Unique risk of bone marrow transplant recipients. Am J Med 1987;83:709-18.

28. Salkin IF, Martinez JA. Opportunistic infection of the spleen caused by Aureobasidium pullulans. J Clin Microbiol 1986;23:828-31.

29. Kwon-Chung KJ, Bennett JE. Medical mycology. Philadelphia: Lea \& Febiger; 1992.

30. Lopes JO, Alves SH, Benevenga JP, Brauner FB, Castro MS, Melchiors E. Curvularia lunata peritonitis complicating peritoneal dialysis. Mycopathologia 1994;127:65-7. 